\title{
VIRTUAL MUSEUMS FOR LANDSCAPE VALORIZATION AND COMMUNICATION
}

\author{
E. Pietroni \\ CNR Institute of Technologies Applied to the Cultural Heritage, via Salaria km 29,300 00015 Monterotondo st. (Rome), Italy - \\ eva.pietroni@itabc.cnr.it
}

Commission II, WG VI/4

KEY WORDS: Landscape representation, Digital ecosystems, Virtual museums, Digital storytelling, Embodied Interaction, New media, participatory approach.

\begin{abstract}
:
Research in the domain of landscape virtual reconstructions has been mainly focused on digitization and recording inside GIS systems, or real time visualization, paying a minor attention to the development of a methodological approach for the landscape narration, combing different registers, conceptual, emotional incitements and, thus, able to arouse in the public a feeling of emotional "sensing" and self- identification. The landscape reflects also the human activities in the territory and the communities' cultural patterns, their sense of "belonging". In a virtual museum of landscapes, the multidisciplinary approach, the multiplication of perspectives and voices, storytelling, acquire primary importance. A Virtual Museum of landscapes should integrate both holistic and delimited visions. The holistic vision requires a diachronic approach, including both present and past phases of life. On the other side, delimited, or "monographic", representations are useful to go deeper into specific and exemplar stories, regarding specific groups of people.

Beside, the emergence of new social media enhancing cultural interactions among people induce the creation of specific social platforms for Cultural Heritage for the active participation of a large number of stakeholders. Co-creation scenarios and tools can be particularly promising. Aton is an example of front-end VR social platform in the web end, for the efficient streaming of medium/large landscape, their exploration and characterization.

The Tiber Valley Virtual Museum is an example of sensorial cultural landscape. Starting from the acquisition of topographical data through integrated technologies, several multi-sensory scenarios have been created, inside which visitors can feel embodied and
\end{abstract} involved.

\section{THE LANDSCAPE AND ITS VALORISATION}

\subsection{Concept of landscape}

With the European Landscape Convention, adopted by the Council of Europe in 2000, some key concepts were defined:

a) the primary objective of heritage protection is to improve the quality of the landscape; b) the landscape is extended to the whole territory; c) the active role of the people in the identification of the landscape is renewed and highlighted.

The definition of the landscape concept is as follows: "A certain part of the territory, as perceived by the people, whose characteristics derive from the action of natural and / or human factors and their interrelations." (CEP Article 1 (a). (http://www.coe.int/en/web/landscape)

Therefore the Convention does not consider the landscape as a purely objective phenomenon (such as the territory for example) nor as purely subjective (the landscape as a state of mind), but rather as something that consists in the interaction between the two sides. Landscape is not only the background of men's actions, it is a living reality that is constantly changed by these actions, thus assuming always different features and new meanings. It becomes a reflection of the cultural identity, as its evolution is guided by the economic mechanisms and sociocultural values that govern the action of a certain society and by the universe of signs and symbols to which it gives meaning. The landscape, as the result of such interactions, is present everywhere and not just in "certain places". Therefore the landscape is the mirror of our being.
Designing a virtual museum of the landscape means to define a methodology of valorisation and enjoyment of the territory interpreted as an ecosystem, a context of past and present relationships, geographic, historical and anthropological reality settled in specific forms, knowledge, memories, behaviours, cultural and artistic expressions.

The virtual museum of a landscape should be not only a showcase for outdoor visitors, but it should also welcome the expression of an active and participatory local community. A place where citizens can understand their story and identity to improve the quality of their present environment and life.

Today peripheral territories, especially close to the great urban attractors, live almost always a condition of marginality, although they are often rich of historical and archaeological evidences and of great natural value. Since the Second World War, in many European countries small historic villages upon the hills, mostly of medieval origin, are experiencing a gradual depopulation in favour of the big cities, and this process seems to be inexorable in the coming decades, until the definitive abandonment. Territory is often travelled or lived with a low awareness, and this causes neglect or disinterest.

The translation of the cultural heritage in "virtual heritage" is a process that recapitalises, disseminates and spreads its value, opening opportunities for a new economy. The capital resides in cultural information and communication, enhanced perception and interpretation, thus new potential economies can be activated in the territory, new consumption and tourist flows can be solicited, and more advanced cultural interactions (Forte and Campana, 2016). In fact through the virtual we can re-establish the network of relationships that connect the cultural object / 
site / landscape to its context of life, the anthropological, historical, philosophical and social themes.

\subsection{Musealising the landscape through virtual technologies: state of the art and suggested criteria.}

Some key questions need to be taken on: how can we conceive and create a virtual museum of landscapes? Which is the best epistemological approach and the proper methodology? Up to now these aspects have not been investigated in detail or by pursuing multidisciplinary and coherent criteria, considering also the rapid grow of new models of social participation (internet, social media, etc.). At international level, the epistemological approach for the virtualization and communication of landscapes has still little attention and is not consolidated. The V-MusT.NET project itself, (the European Transnational Network on Virtual Museums, (www.v-must.net, FP7-6-2009 V-MusT.NET - N.o.E., 2011-2015) focused more on collections of artefacts or archaeological sites, dedicating very little attention to the landscape.

Powerful technologies for topographic survey and spatial documentation, GIS tools, remote sensing techniques and 3D modelling are commonly used today to elaborate, analyse and represent spatial data regarding landscapes. They can greatly increase the level of knowledge of the landscapes in terms of information, interaction, learning. These tools are very useful for scientists to organize data, but they don't develop a narrative. Landscapes Virtual Museums must link real to virtual world, present and past, scientific approach and individual feeling, many levels of perception and interpretation, multiple communicative registers, voices and perspectives, making understandable the interaction between distinct component of tangible and intangible cultural heritage. Narration, interaction, "embodiment", multi-sensory solicitation are essential elements in creating an experience about landscapes (Turri, 1982). They are directly related to the "emotional intelligence" of man and are crucial in fostering the contact between our sensitivity and the deep essence of cultural heritage, (Ragghianti, 1974), (Panofsky, 1996). As psychologists, neuroscientists and philosophers have recently highlighted, emotions play a decisive role in the creative and intuitive processes of human knowledge (Di Dio et al. 2015), (Gianni Falvo 2013). Emotions motivate understanding, participation, self-identification, internalization and thus a process of memorization and learning. Emotional involvement and participation are therefore of growing relevance in the next generation of virtual museums, promoters of easier and broader social inclusion, without any subtraction of scientific knowledge. Scientific content go hand in hand with art and technology and the blend of the disciplines is essential to create a good communication.

Therefore a virtual museums of a landscape needs a strong link among several fields: geographical, naturalistic, historical, anthropological, technological, artistic and symbolic domains, and between real and symbolic worlds (observed landscape, interpreted landscape, map-scape, reconstructed landscape, mind-scape).

A Virtual Museum of landscapes should integrate both holistic and delimited visions. Holistically, a landscape can be compared to a living organism in continuous evolution: it needs to be considered as a unique context whose identity is much more than the sum of its single parts. This holistic vision requires a diachronic approach, including both present and past phases of life and the consideration of dynamic relations and processes. On the other side, delimited, or "monographic", representations are useful to go deeper into specific and exemplar stories, regarding the life and the way of thinking and behaving of specific groups of people. The identity of the landscape, the "sense of place" arises from the integration of the two levels.

In this perspective, in next future research virtual landscape can become a simulation environment populated by artificial life, an open digital ecosystem where communities of users will interact in a multimodal way. Recent promising trends in information and communication technologies, identify a strong superimposition of different research disciplines, AI (artificial intelligence), VR (virtual reality) and AL (artificial life). The concept itself of "virtual reality" is changing, in the sense of a virtual environment with artificial evolving creatures. According to the ecological thought, relations existing between the organisms and the environment dominate the whole knowledge of both the real and the virtual domain. A living creature is characterized by the capability to auto-reproduce and self organize. Artificial societies and autonomous agents perform behaviours established by themselves in relation with a specific situation happened in the virtual world or on the base of casual effects; in the most advanced applications, agents have different aptitudes and attributes, are able to learn interacting each other or with the humans. They influence each other according to different and variable dynamics and they interact with users generating a succession of unexpected events and actions. The integration between traditional disciplines related to the study of landscapes and simulation algorithms based on artificial life is really crucial if we don't want to limit the work to the 3D representation and visualization of a pre-defined environment (even if at different chronological steps of its evolution), but, on the contrary, our purpose is the simulation of the transformation processes in order to reach a deeper and critical comprehension of it, (Forte et al. 2005).

This perspective supposes some fundamental questions: which are the metaphors to relate human users, artificial societies and cultural contents? Which codes can we share? How can we create and share meanings? How can we elaborate these kinds of information? (Annunziato et al. 2008).

Finally, nowadays, in the digital era, it becomes more urgent to converge research efforts in reconsidering the role of the final end-user and the interaction among visitors. The European Cultural Heritage sector has witnessed the emergence of new technological models which led to the creation of new Social Platforms able to encourage the active participation of stakeholders with diverse backgrounds and a deeper comprehension of the art and cultural themes dealt with by Virtual Museums. By these means, the difference between real and virtual museums tends to vanish and the role of the audience little by little gains importance: not necessary a mere passive viewer or observer, but, most importantly, a creator and provider of data and contents.

Many cultural heritage professionals have been participating in many EU projects with the ambition to create platforms for virtual museums, content management systems and repositories that should help them to develop, create, maintain and integrate digital heritage contents in virtual museums. Many projects promised online platforms/repositories (see list in http://www.vmust.net/library/documents/d41-available-platform-

components-and-platform-specification), competence centers (http://www.vcc-3d.com/) and authoring tools that after some time failed or where not used. The main problem was that often these tools and centers were not properly tested and there was no real business cases developed.

Therefore, the envisaged epistemological approach for landscapes virtual museum will:

1. Implement interconnection of TERRITORIES, STORIES, COLLECTIONS (museums of the territory) and USERS; creating many potential 
different scenarios reflecting personalisation and recommendation of the user(s).

2. Have a MULTIDICIPLINARY APPROACH, involving expertise in history, geology, archaeology, architecture, urban and environmental planning, literature, arts and ICT.

3. Have an IMPROVED VISUALIZATION. Projection Mapping, Virtual and Augmented Reality, Mixed Reality through the use of Virtual Set and advanced Compositing techniques, Holograms, Interactive videos, etc. will help the immersive experience of the user.

4. Let people access to digital contents in different moments of their cultural experience (before-duringafter the visit) through a "MULTICHANNEL" approach (online - onsite - mobile) and a wide range of applications.

5. Include curatorial sections and contents and, besides, be open to external contributions, allowing CITIZENS' INCLUSION and PARTICIPATION through specific authoring tools.

6. Follow a SCIENTIFIC APPROACH with an improved access to METADATA, allowing storage, update and re-use of digital assets in order to make them available for further projects.

7. Be based on modularity, SCALABILITY, transmedia, interoperability and sustainability of the digital platforms.

8. Present new ways of PERSONALIZED STORYTELLING, interactivity and adaptive guidance in order to support the public and not make them disoriented in complex virtual environments.

Develop links within the people of the territory taken in consideration, and possibly suggest a wider connection with an even wider context

\section{PARTECIPATORY PROCESSES}

\subsection{Social platforms for the co-creation of virtual landscapes}

The emergence of new social media enhancing cultural interactions among people induce the creation of specific platforms for Cultural Heritage that encourage an active participation of a large number of stakeholders. The new challenge, for cultural heritage professionals, is to transform the traditional and static one-to-many knowledge transfer model, used until now in traditional academic communication channels, with a dynamic and interactive many-to-many communication model. There is an increasing request of digital frameworks open to the communities for the accessibility, study, participatory and sustainable management of cultural resources and assets. In the case of landscape characterization co-creation scenarios and tools can be particularly promising. The potential of web communities in 3D virtual environments is very high in terms of embodiment and cognitive impact: users can perceive, meet, dialogue, touch objects, transform the surrounding virtual environment, exchange information, make and simulate hypothesis, perform actions following a common a purpose. Knowledge comes from "enaction" and it is built essentially on sensory motor skills and direct experience in the $3 \mathrm{D}$ cyberspace. Cognitive activity is "embodied", not separated from the sensorial perception (Mellet-D'Huart 2006), (Varela et al. 1991).

A pioneer example of 3D multi-user virtual domain in the web came from The Integrated Technologies of robotics and virtual environment in archaeology project (2006-2009), financed by the Italian Ministry of the University and Scientific Research. In this case CNR ITABC realized a 3D real time platform for the web addressed not to the general public but to the community of archaeologists. This collaborative environment was conceived as a space of simulations and education, an open laboratory allowing experts to share and exchange cultural contents and interpretations. They could load a 3D scenario, related to an archaeological site or landscape, meet, chat and interact in real time, share data. Each scene could be edited by the users: it could be disassembled, changed and recomposed again, models could be added, hidden, moved, modified according to different hypothesis. It was possible to add link to new metadata, save new versions of the scene, share it and discuss within the community. Users became creators of ideas and contents, establishing relationships with other users (Pietroni et al. 2009). The framework was developed in Virtools DEV and it required a plug in to be accessed in the web.

Similarly, the Virtual Rome project developed by CNR ITABC (2007-2009), was an Open Source web VR project, based on geospecific data, with front-end (VR webGIS) and back-end frameworks for the interpretation, reconstruction and $3 \mathrm{~d}$ exploration of the archaeological and potential landscape of ancient Rome (Pescarin et alii, 2008). The purpose was the creation of a on line $3 \mathrm{~d}$ environment, embedded into a webbrowser. The back-end framework was conceived as a collaborative platform: through a 3D Content Management System, different professionals could be involved in the activity of landscape reconstruction.

\subsection{The Aton platform}

Starting from these premises the Aton platform is at the moment under development at CNR ITABC since 2013 (http://osiris.itabc.cnr.it/scenebaker/index.php/tag/webgl/).

Aton is a flexible web front-end (PHP + JavaScript) allowing to publish and interactively explore multi-resolution $3 \mathrm{D}$ assets and scenes online through modern browsers (both desktop and mobile). Aton has been enriched by Landscape Services, already developed for the ARIADNE project (funded by the EC under the FP7 scheme; Ref : FP7-INFRASTRUCTURES-2012313193) that are a set of responsive web services including large terrain datasets generation, 3D landscape composing and 3D model processing. It leverages on powerful open-source frameworks and toolkits such as GDAL, OSGjs, OpenSceneGraph and ownCloud, (A. Antal et al. 2016).

Aton has been specifically conceived for: a) medium-large landscape 3D visualization and exploration in real time, through an efficient streaming algorithm; b) co-creation processes, involving experts, citizens, students.

The use of modern PBR (Physically Based Rendering) approaches, re-adapted to fit WebGL world and multi-resolution landscapes, provide aesthetically pleasing experiences inside a common browser. The presentation layer is able to scale down and tune itself automatically according to the device that is being used, in order to maintain the interactivity also on mobiles.

Aton allows also spatial contextualization of multimedia contents through a powerful 3D annotations system with HTML 5 contents. These contents are integrated by means of an editor based on WYSIWYG "What You See Is What You Get" approach. Such an editor is a system in which contents (text and graphics) appears, during editing, in a form that is very closed to its appearance when printed or displayed as a finished product. Registered users can annotate and enrich the 3D landscape with several multimedia contents (formatted text, images, audios, videos, etc.). The usage of a built-in editor and 
WYSIWYG approach provides an easy-to-use interface to insert contents and enrich current 3D landscape. It is possible to include also 3D discussion streams (chat-like approach) to further boost the social aspect within current 3D landscape, expanding it with an additional layer of comments (fig.1).
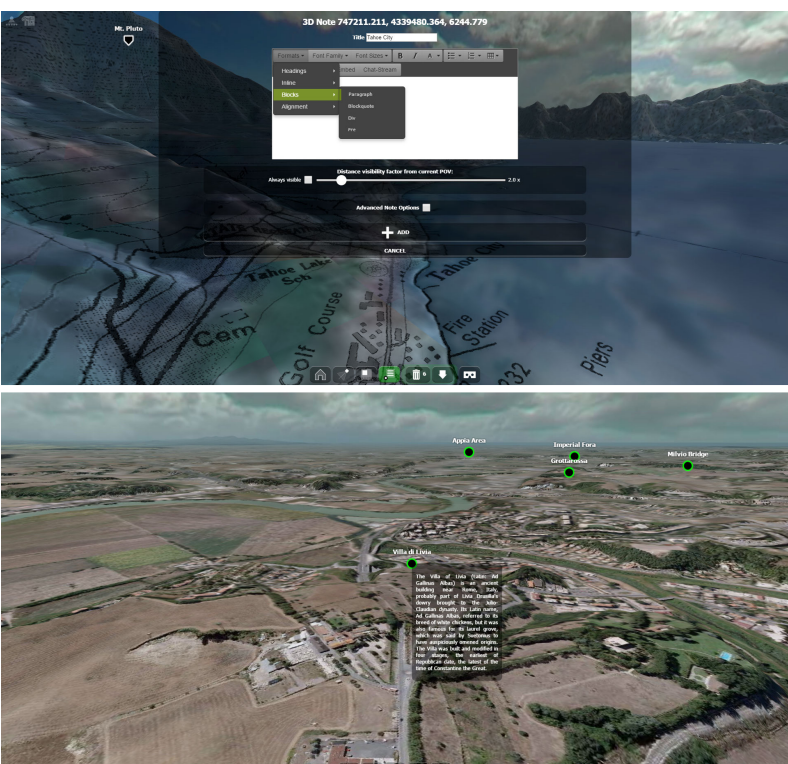

Figure 1. Aton interface and editor to insert 3D annotations

This means that every user can:

1) Use this framework to generate new 3D landscapes (starting form GIS data previously elaborated) that can be saved in many different format (i.e. .obj, .max formats to be used elsewhere or webGL to be visualized in real time in the same browser);

2) Add and contextualize new contents in the existing 3D landscapes: monuments, settlements and features; these contents can be geo-referenced or associated to specific places through HTML5 3D annotations. A 3D annotation can include every HTML content (texts, images, videos, audio files, google maps/google earths, chat, other 3D WebGL pages, live streaming pages, spherical panoramas, panoramic videos, etc.).

3) Add or save Points-of-View (POV) as annotations, save and share the current scene + POV through permalinks.

4) Navigate across annotations (exploring interactive storytelling or specific contents) save and share them.

Given the huge quantity and typology of contents that the users can upload on the platform, tools for indexing and filtering of 3D annotations are under development. In this way the user will decide what kind of contents should be visualized and experienced on the territory during the fruition (narrations, videos, archaeological contents, geological information and so on). Moreover, 3D annotations in the 3D landscapes could be characterized by different colours according to their typology/subject (white for 3D bibliography, brown for geological data, red for narratives and poetries, blue for music, green for ecosystem, orange for semantics and metadata, pink for reliability levels and so on). The Application Program Interfaces of this platform are still under improvement to allow integration with external web services such as the optimization and automatization and of some processes: reduction of polygonal density in the case of 3D models, resize of textures and images, export of 3D models and images in other file formats, creation of LOD (Level Of Detail).

These potentialities allow a wide audience to add contents, cocreate landscape and scenarios that could be inconvenient if external contents are not regulated; to avoid addition of not appropriate content different rights of editing and validation for different users will be created.

\section{SENSORIAL LANDSCAPES.} THE VIRTUAL MUSEUM OF THE TIBER VALLEY

The Virtual Museum of the Tiber Valley is an example of sensorial cultural landscape.

Finaced by ALES-Arcus S.p.A, it has been developed by CNR ITABC in 2011-2015, in collaboration with the Italian Ministry of Cultural Heritage. The territory taken in consideration is an area North of Rome extending for $60 \mathrm{x} 40 \mathrm{~km}$, crossed by the Tiber River and by three ancient Roman roads, via Salaria, via Flaminia and via Tiberina.

Starting from the acquisition of topographical data through integrated technologies, photo interpretation, GIS systems, multi-scale representations (landscape, site, intra-site), several many scenarios have been created.

The observable archaeological landscape continuously alternates with the multiple projections of its potential reconstructions of the past (reconstructed landscape), or imaginary dimensions (mind-scape), (Bateson, G., 1972), (Forte, 2003).

\subsection{User experience design}

This project explores a new approach in the virtual landscape exploitation and communication, trying to combine and integrate different languages and paradigms: virtual reality, gesture-based interaction interfaces, cinematographic and theatrical techniques, virtual set practices, gaming dynamics, augmented reality.

On site installations and on line multimedia contents have been developed, for the different moments of the users' cultural experience.

On site multimedia applications are accessible both in Roman and in peripheral museums; among them a VR application characterized by gesture-based interaction has been created for in the National Etruscan Museum of Villa Giulia where is can be accessed since December 2014.

The VR application is visualized on three aligned 65 " screens, arranged in a semicircle, in order to arouse a feeling of immersion and perceptive involvement. It consists of four scenarios, (video demo: https://vimeo.com/129867454) that the user can explore migrating among different "avatars":

1. "On the spirals of the Tiber: the landscape of the origins": the user can fly, like a bird (using his arms), over an evocative 3D representation of the middle Tiber valley landscape.

The representation of the landscape is based on a accurate digital DEM and the most important places are put in evidence, but the rendering is not photorealistic; on the contrary it aims at stimulating the imagination of the visitor following a symbolic approach; 3D graphics resemble a game, the sounds have been composed starting from ethno-musicological studies, redeploying traditional popular sounds and folk songs documented in the Roman countryside in this area (fig.2).

Crossing magic circles in the sky the user can travel back in time and activate videos related to:

- the geological and geomorphological evolution of the territory at different stages, from 3 millions years ago until today,

- a potential reconstruction of the landscape in the VIII-VII century $\mathrm{BC}$, telling about the birth of the cities from the astonished perspective of men and women attending the fast transformation of their world. 
These movies present several 3D reconstructions, beside real images and video shots of the actual landscape.

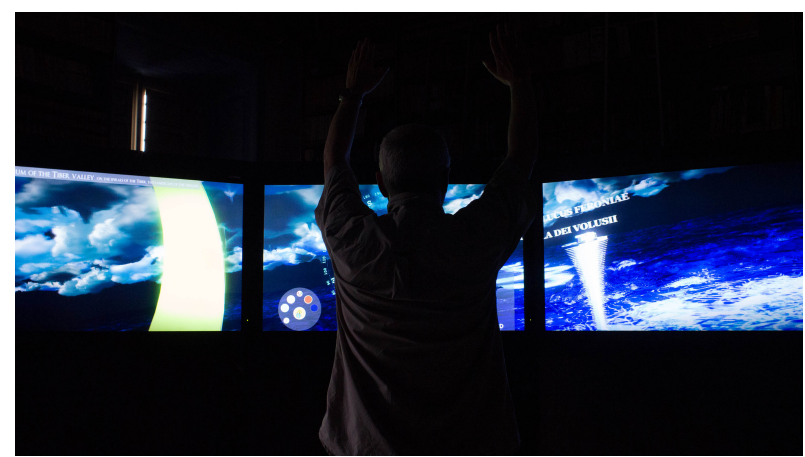

Figure 2. Virtual Museum of the Tiber Valley: aerial scenario

2. "In the setting of the sun the Tiber gleams. The secrets of the river: swimming underwater in the current of the Tiber, like a fish, the visitor can experience the memory of the river; he meets fluctuating images, iconographies, sounds, literary fragments taken from ancient and contemporary poets and authors. He can swim freely, following these dynamic memories or he can awake them touching fragments of painted walls collapsed in the river. Literary quotations are spoken by a multitude of voices. The visitor meets other fishes living in the river whose movements are controlled by artificial intelligence and swarm dynamics (fig.3).

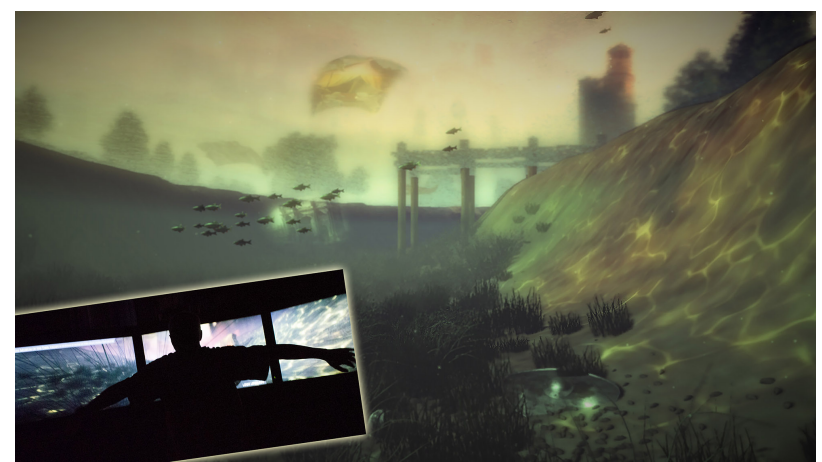

Figure 3. Virtual Museum of the Tiber Valley. Underwater scenario

3. "Mena's story, Volusii's Villa": the user is involved in an immersive experience inside this roman Villa. At the begin he can see how the villa looks like today (in a movie), then he is brought back in Augustan time, in the 3D reconstruction of the Villa at its maximum richness, and he can start the real time exploration, (fig.4). The user acts like a man walking through the space. Here he is involved in the personal story of Mena, a freed slave living his own psychic drama. This character is imaginary but plausible because his profile has been created following several literary and historical sources related to freed slaves' condition during Augustus' time and it is also historically connected to the presence of thousands of slaves working in the huge extensions of land owned by the Volusii around the villa. The archaeological and historical context is thus used as scientific background of this engaging story.

The user can relax following a predefined camera path (virtual steadicam) while Mena's monologue develops, but he can decide to stop in every moment and look around to analyse details of the architecture or decoration. In some places he finds crossroads and he can decide in which direction he wants to go on, to follow a specific part of the story (interactive storytelling).

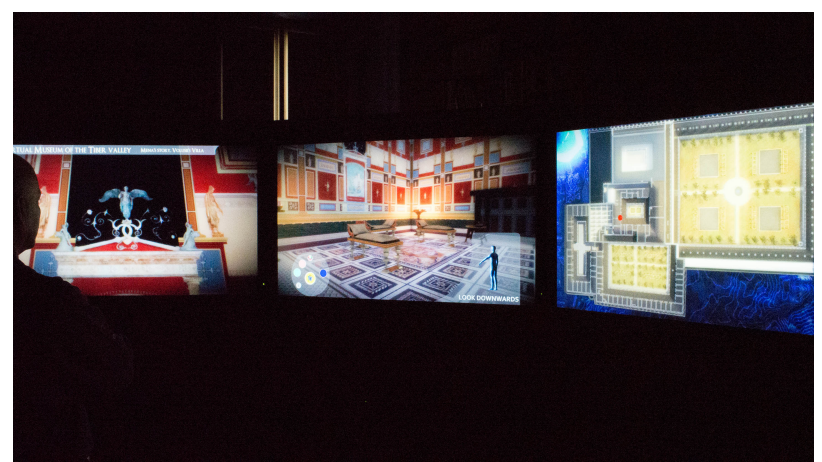

Figure 4. Virtual Museum of the Tiber Valley. Volusii's Villa scenario

4. "Here only you can see me. Lucus Feroniae“: the user follows a predefined path through the ancient Roman settlement of Lucus Feroniae reconstructed in 3D during Tiberius' and Trajan's time. As in Volusii's Villa, he finds crossroads, corresponding to specific narratives, and he can choose the direction he prefers to enter the different stories and places.

Real actors have been filmed on a green screen and then integrated in the virtual scene to represent the ancient characters performing their daily activities in the reconstructed town; they dialogue and interact among them and occasionally with the user. The memory of the ancient Italic goddess Feronia is also evoked: even if no longer worshiped in Roman times, her sanctuary was a point of attraction for many people of Center Italy in the archaic period. Only Cesia, a little child, is able to see the goddess and hear her voice (fig.5). Thanks to the magic power of Feroniae we are able to see how the city of Lucus will be transformed one hundred years later.

During narratives the visualization is a cinema-like: a unique large viewport distributed on the three screens. On the contrary, during the exploration three different viewports are shown on the three screens to compare the current archaeological site to its 3D reconstruction: on the left and central screens the observed archaeological environment and the 3D reconstruction of the past structures are shown in parallel, from the same points of view, in order to offer the visitor an "augmented" perception of the space and a better comprehension of the site (fig. 6).

On the right screen, a $3 \mathrm{D}$ perspective view of the whole city is shown with the evidence of the user's position during his exploration. This visualization is useful to let the user a better orientation. The multiplication of the points of view (first person, third person, present, past) and narrative perspectives stimulates the user's active and critical participation within the virtual environment.

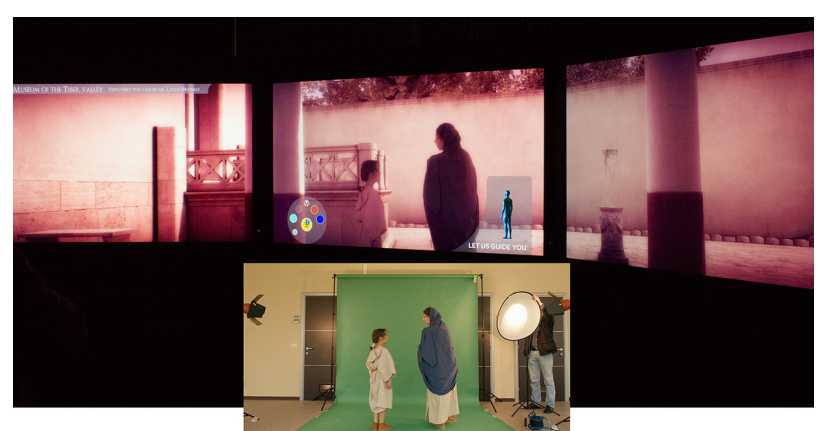

Figure 5. Virtual Museum of the Tiber Valley. Lucus Feroniae scenario, mixed reality. 


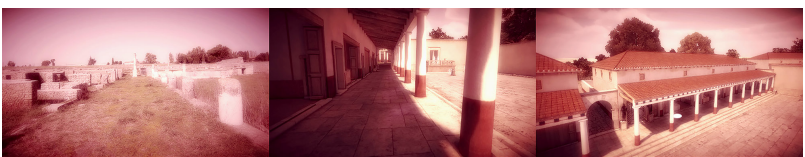

Figure 6. Virtual Museum of the Tiber Valley, Lucus Feroniae scenario. Observed and reconstructed landscapes are compared

Each scenario needs from 5 to 10 minutes to be enjoyed. The user is involved in that flow and rhythm, his experience is multi-sensorial and emotional.

The application needs to be installed in a closed and silent space/room, because under this conditions the public can concentrate on storytelling and evocation and thus can feel really immersed in the experience.

The user can interact through body movements, thanks to the use of markless sensors for motion capture available on the market since 2011, such as Microsoft Kinect (Pietroni and Adami, 2014). One person at a time can guide the system in the interactive area in front of the screens $(4 \times 4 \mathrm{~m})$. The other users (about 15 persons) can watch seating or standing in the space all around but they can alternate in every moment in the active role. The alternation between different phases of passive enjoyment and active interaction have been used, with the aim to create rhythmic variations making the experience not too difficult, not repetitive and boring but always unexpected and exciting, thus more attractive and relaxing, for a wide audience.

Storytelling establishes the need of a precise "direction", also in case of an interactive application and a virtual reality environment. To structure this "direction" novel approaches and communicative formats have been created mixing techniques coming from different disciplines and domains.

The interaction mode through the body gestures enriches the attractiveness of the installation, the feeling of embodiment, the active participation and the sensorial immersion inside the landscape (Pietroni and Adami, 2014).

The engagement inside the stories comes not only from the script, but also from the visual moods, the colours, the general atmosphere, the lights and soundscapes, the camera movements, that produce another layer of communication. This subliminal layer, in particular, based on emotions and perceptions represents the immersive part of the experience (Ryan, 2001).

At the same time it has been necessary to find an harmony among all these inputs and the right time of use, according to the location of the multimedia application inside the museums. Specific evaluation studies regarding the impact of this project on the public have been carried on following a precise protocol, and already published (Pagano et al., 2016), (Pietroni et al., 2016).

In the website (www.museovirtualevalletevere.it) the users can find all the contents that have been integrated in the on site VR ad multimedia installations together with additional movies telling the story, the activities, the "vision" of persons living in the territory and interviews to experts regarding different aspects of the landscape. Some documentaries that we have produced for the national Italian television are accessible.

It is also possible to find several materials regarding the creation process and the project's backstage. In the Download sections every user can freely access to scientific documentation, data coming from the topographical survey and GIS, interpretative studies, screenplays, reports, papers and presentations regarding the different aspects of the project, user experience studies, together with the results of evaluations that have been conducted on a differentiated public (general users of the museum or students of secondary schools).

\subsection{From the map-scape to the reconstructed landscape.} A methodological proposal

In this paragraph the methodological approach followed for the $3 \mathrm{D}$ reconstruction of the potential ancient landscape of the medium Tiber Valley in the Orientalising (VIII-VII century BC) ad in Roman (I BC- II century AD) periods will be presented. A complete pipeline procedure, from GIS data elaboration up to final realistic 3D renderings and communication has been developed (Pietroni et al., 2013). Several kinds of information have been combined: geological and ecological data, historical, archaeological and demographical studies. As a first step a the digital elevation model (DEM) of the actual landscape coming from Istituto Nazionale di Vulcanologia, (resolution $10 \mathrm{~m}$ ) has been used and textured with a satellite image (IRS, resolution 5mt).

Secondly another DEM has been generated from aerial photogrammetry (resolution $5 \mathrm{~m}$ ). A set of 12 aerial IGM analog photos has been used, taken in 1954, before the construction of the two major modern infrastructures: the dig of Nazzano (1955) and the highway (1961). Since we can assume that in the Orientalising and Roman periods the geomorphology was quite similar to the one we can observe today, this DEM constituted the base for the $3 \mathrm{~d}$ model of the terrain in the past ages. It has been coloured and characterized in a realistic way through 3D and 2D graphic libraries.

Basically, for the creation of the past ecosystems the archaeological map and the land unit map (pedo-landscape) were the starting points. The latter was useful to define the soils composition and their attitude to host specific ecosystems, both natural and cultivated.

From the original 75 land units, 6 main macro-ecosystems have been obtained after a process of simplification (eco-landscape): 1) volcanic; 2) sedimentary (sandy-conglomeratic-clayish); 3) calcareous of low-medium height; 4) calcareous of considerable height; 5) valley eco-landscape (lower terraces included); 6) Tiber banks eco-landscape.

The geomorphology and geology of the territory, the slopes, the orography, the accessibility of the river have been also analysed, and an hypothesis of ancient courses for the Tiber has been elaborated, together with the possible presence of ancient harbours or crossing points/structures on the river. The results were compared with the archaeological remains and we found good correspondences.

Regarding the definition of the potential natural vegetation for each macro-ecosystem, the existing thematic cartography elaborated by Regione Lazio and Provincia of Rome has been taken in consideration. An ecosystem consists in a specific mixed set of plants that adapt to that soil, climate, orientation and so on. The combination and disposal of the plants in each ecosystem is natural or linked to the cultivation techniques existing in that specific age. We can assume that the potential natural vegetation in the past was not substantially different from today. These data have been combined with the archaeological map and demographic analyses for the Orientalising and the Roman periods, in order to define the areas influenced by the presence of human settlements and activities ("buffers").

A specific methodology has been followed for the buffer creation. Several factors have been considered, in relation with the geography and geomorphology of the territory, the distance of the cultivated lands from the villages or town, from the infrastructures (roads) and from the natural resources in the landscape (rivers, etc.), the economic model, the technological level and the potential movement of the population in the daily conduction of their work. 
Each one of these factors has a certain level/proportion of importance in relation to the others, and this relation can change through the different historical ages. The existing bibliography about demography, settlement patterns, alimentation and food in the past ages, together with information coming from pollen analyses and archaeology supported the characterization of the cultivations and vegetal species spread on the landscape.

The final result of this work has been the elaboration of a colour map that describes the past landscape, including natural and anthropic areas. Each color of this map represents a specific ecosystem.

The methodological process followed for the Orientalising and Roman periods can be easily replicated for other chronological phases, changing the weight of the single parameters.

The final step has been the translation of this symbolic description of the landscape (GIS) in a realistic 3D representation, through powerful tools for landscape generation and design. The final representation, aimed also at cultural communication to the public, is of two types: a real time application developed in Unity $3 D$ and movies realized in Vue (fig. 7). Data have been processed and optimized differently for the two outputs.

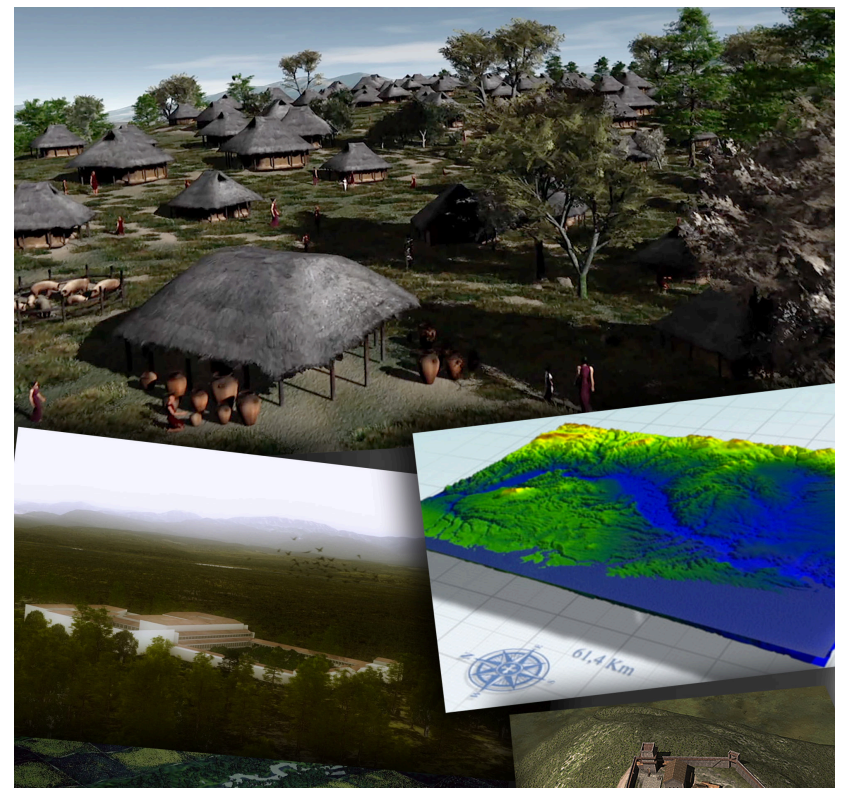

Figure 7. Virtual Museum of the Tiber Valley. 3D reconstruction of the potential landscape in its geological evolution, in the Orientalising and Roman periods.

Beside the landscape, specific archaeological Roman sites have been documented and reconstructed in 3D. These sites have been documented and represented in 3D through activities of survey, laser scanning, UAV, photogrammetry and structure from motion (Bottom-up approach). 3D reconstructions in Augustan and Trajan periods have been realized adopting a proven methodology and a scientific approach based on archaeological evidence, literary documentation and typological comparisons (Top-down approach) (Pietroni et al. 2015)

\section{CONCLUSIONS}

Sensing and emotions are fundamental in the life experience, in self-consciousness processes and they are the engine of knowledge and development of the individuals. "Sensing" a cultural landscape means also the capacity to enter in contact with those elements that let us "recognize" something and move our emotions.
In the Tiber Valley Virtual Museum the observable archaeological landscape continuously alternates with the multiple projections of its potential reconstructions of the past (reconstructed landscape in different ages), or imaginary dimensions (mind-scape) (fig.8). Non linear interactive storytelling combines different languages: virtual reality paradigms, gesture - based interaction interfaces, cinematographic and theatrical techniques, virtual set practices, augmented reality, going beyond the traditional descriptive and academic style. The methodological approach followed for the reconstruction of the potential ancient landscape and for its narration has been presented.

On the other side in the next future we have to deal with a very ambitious technical challenge: to create a social platform for virtual museum of landscapes, intended as an open digital ecosystem where communities of users will interact in multimodal way. This means that a landscape is an ecosystem that can be virtually and variously approached, becoming scenarios of different simulations and interactions, where the user can perform activities and create a "visual drama" beyond what they can see.

Such a methodology can produce a great impact on education, as it brings cultural transmission to a higher level of understanding, capable of awakening and strengthening in the communities their sense of belonging. The landscape virtual museum becomes a mirror of our cultural identity favouring a major respect for our living environment.

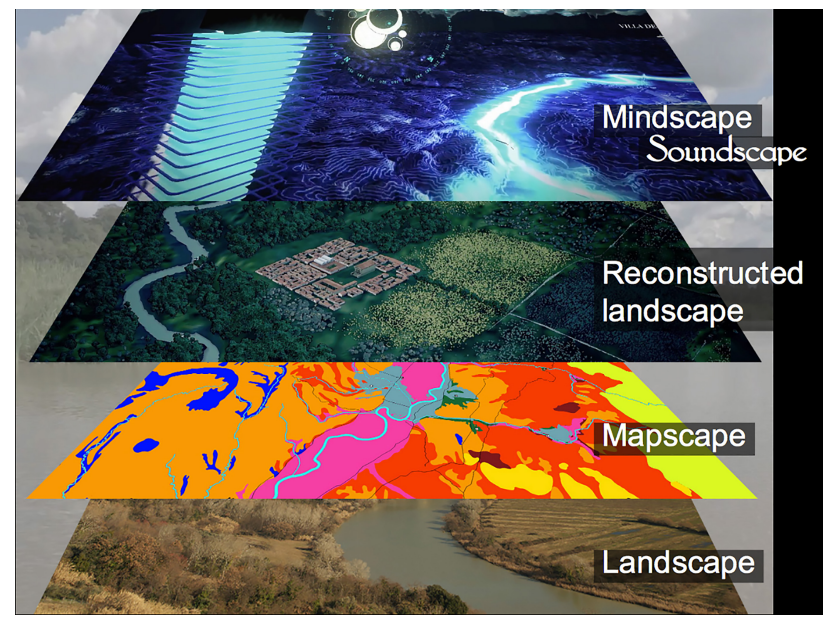

Figure 8. Landscape virtual museum. Levels of representation

\section{ACKNOWLEDGEMENTS}

Acknowledgements to: ALES-Arcus, Soprintendenza Archeologia, Belle Arti e Paesaggio per l'area metropolitana di Roma la provincia di Viterbo e l'Etruria Meridionale, Museo Nazionale Etrusco of Villa Giulia who supported the Virtual Museum of the Tiber Valley as Institutions, E.V.O.CA. srl and Franz Fischnaller who contributed to the realization of the project, sharing ideas and concretely developing the applications, Antonia Arnoldu -Huyzendveld for the geological consultant in the reconstruction of the ancient landscape of the Tiber Valley, Bruno Fanini who is developing the Aton platform at CNR ITABC. 


\section{REFERENCES}

Annunziato, M., Bonini, E., Pierucci, P., Pietroni, E., 2008. Cultural mirrors: an epistemological approach to artificial life for cultural heritage communication. In: Digital Media and its Applications in Cultural Heritage, edited by Jamal Al-Qawasmi, Michele A. Chiuni, sabry El- Hakim, pp. 219- 135.

Antal A., Bota, E., Ciongradi, C., D’Annibale, E., Demetrescu, E., Dima, C., Fanini, B., Ferdani, D., 2016. A complete workflow from the data collection on the field to the deployment of a Virtual Museum: the case of Virtual Sarmizegetusa. In EUROGRAPHICS Workshop on Graphics and Cultural Heritage. Genova, C. E. Catalano and L. De Luca (Editors). DOI: 10.2312/gch.20161386 ).

Bateson, G., 1972. Steps To An Ecology Of Mind. San Francisco, Chandler Press.

Di Dio, C., Ardizzi, M., Massaro, D., Di Cesare, G., Gilli, G., Marchetti, A., Gallese, V., 2015. Human, Nature, Dynamism: The effects of content and movement perception on brain activations during the aesthetic judgment of representational paintings. In Frontiers in Human Neuroscience. January 2016. DOI: 10.3389/fnhum.2015.00705. eCollection 2015.

European Landscape Convention, 2000-2010. Programme by the Council of Europe, http://www.coe.int/en/web/landscape

Forte, M., 2003. Mindscape: ecological thinking, cyber anthropology and virtual archaeological landscapes. In: The Reconstruction of Archaeological Landscapes through Digital Technologies, ed. M.Forte, P. Ryan Williams (eds.). Oxford Archeopress (BAR International Series S 1151), pp.95- 109

Forte, M. and Campana, S., (edited by), 2016. Digital Methods and Remote Sensing in Archaeology. Archaeology in the Age of Sensing. Springer International Publishing, Series title "Quantitative Methods in the Humanities and Social Sciences". eBook ISBN 978-3-319-40658-9, DOI 10.1007/978-3-31940658-9, Hardcover ISBN 978-3-319-40656-5, Series ISSN 2199-0956

Forte, M., Pescarin, S., Pietroni, E., 2005. The Appia Antica Project. In: The reconstruction of archaeological landscapes through digital technologies, Bar Int. Series 1379, 2005, pp. 7992.

Gianni Falvo, P., 2013. La Madonna del Cardellino di Raffaello Sanzio; realizzazione di un filmato del restauro ad alta definizione. In: Storicamente, Art. N.2, Issue N.9 - 2013, ed. ArcheotipoLibri.

Pagano, A., Pietroni, E., Poli, C., 2016. An integrated methodological approach to evaluate virtual museums in real museum contexts. In: Proceedings of the 9th annual International Conference of Education, Research and Innovation, 14-16 November, 2016, Seville, Spain, pp. 310-321. ISBN: 978-84-6175895-1, ISSN: 2340-1095. Doi: 10.21125/iceri.2016.1077.

Panofsky, E., 1996. Il significato nelle arti visive, Einaudi.

Pescarin S., Calori L., Camporesi C., Di Ioia M., Forte M., Galeazzi F., Imboden S., Moro A., Palombini A., Vassallo V., Vico L., Back to 2nd AD A VR on-line experience with Virtual Rome Project, The Eurographics Association, 2008.

Pietroni, E., Adami, A., 2014. Interacting with Virtual Reconstructions in Museums: The Etruscanning Project. In
Journal on Computing and Cultural Heritage (JOCCH), Special Issue on Interacting with the Past, Vol. 7, Issue 2, Article $\mathrm{N}^{\circ} 9$, July 2014, ACM, New York, NY, USA, DOI 10.1145/2611375.

Pietroni, E., Ferdani, D., Palombini, A., Forlani, M., Rufa, C., 2015. Lucus Feroniae and Tiber Valley Virtual Museum: from documentation and 3D reconstruction, up to a novel approach in storytelling, combining virtual reality, theatrical and cinematographic rules, gesture-based interaction and "augmented" perception of the archaeological context. In: Proceedings of 43rd Computer Applications and Quantitative Methods in Archaeology Conference CAA 2015, Siena.

Pietroni, E., Pagano, A., Poli, C., 2016. Tiber Valley Virtual Museum: user experience evaluation in the National Etruscan Museum of Villa Giulia. In: Proceedings of WSCG 2016, Plzen, Czech Republic, May 30 - June 3, 2016, ed. By Vaclav Skala University of West Bohemia, Czech Republic, UNION Agency. ISSN 2464-4625 (CD-ROM), ISSN 2464-4617 (print).

Pietroni, E., Palombini, A., Arnoldus-Huyzendveld, A., Di Ioia, M., Sanna, V., 2013. Tiber Valley Virtual Museum: 3D landscape reconstruction in the Orientalising period, North of Rome. A methodological approach proposal. In: Digital Heritage International Congress Proceedings 2013, IEEE Advanced Technology of Humanity, ISBN: 978-1-4799-3169-9, Marseille France; Vol II pp. 223-331.

Pietroni E., Rufa, C., Forte, M., 2009. Embodied virtual communities: a new opportunity for the research in the field of cultural heritage. In: International Archives of Photogrammetry, Remote Sensing and Spatial Information Sciences, Vol. XXXVIII-5/W1 ISSN 1682-1777.

Turri, E., 1982. Dentro il paesaggio, Bertani, Verona.

Ragghianti, L., 1974. Dall'arte al museo, Vallecchi, Firenze.

Ryan, ML., 2001. Narrative As Virtual Reality: Immersion and Interactivity. In: Literature and Electronic Media (Baltimore and London: Johns Hopkins University Press, 2001), Hb, 399pp, ISBN 0-8018-6487-9

Varela F., Thompson E., Rosch, E. 1 991. The Embodied Mind. Cognitive Science and HumanExperience, MIT Press, Cambridge. 